\title{
Biotic Relationships between Plants and Birds at Damietta Coastal Area, Egypt
}

\author{
G.A. Abd-Allah ${ }^{\mathrm{a}}$, M.S. Serag ${ }^{\mathrm{b}}$, N.E.R. El Bakary ${ }^{\mathrm{a}}$, S.G. Habib ${ }^{\mathrm{a}^{*}}$ \\ ${ }^{a}$ Zoology Department, Faculty of Science, Damietta University, Egypt \\ ${ }^{\mathrm{b}}$ Botany Department, Faculty of Science, Damietta University, Egypt
}

*Corresponding author (email: sayedgabr2010@yahoo.com)

\begin{abstract}
The present study aimed to explain the relationships between plants and birds in the different habitats at Damietta coastal area. Field study indicated that one hundred and one plant species were recorded, belonging to 49 families, of these 51 species were wild and 43 species were cultivated. On the other hand, sixty six species of birds were censused belonged to 12 orders and 28 families. Results showed that birds are an essential part of the plant-animal association as they play an essential role in keeping our environment clean and disease-free. Many biotic relationships were found in the present study that conserve ecological balance as birds provide plants with many benefits such as 1) Seed dispersal which play an essential role for the propagation of many plants. 2) Protection as cattle egret, Egyptian barn swallow, black-winged kite, hooded crow, red breasted flycatcher and hoopoe protect plants from insects and earth worm. 3) Pollination as many species of plants, most commonly with red flowers, are pollinated by birds. Furthermore plants provide birds with a number of resources, including food, shelter and protection, nesting material and nest sites. Plants provide food for birds both directly and indirectly. Directly as many species of plant provide a nectar, fruit, seeds and grains to birds for example house sparrow mostly feeds on the seeds of weeds and grasses. It prefers oats and wheat. Indirectly as plants provide habitat for insects which in turn are eaten by birds (e.g. Egyptian barn swallow, Red breasted flycatcher, European barn swallow and European bee-eater). The study showed that vegetation was often densest in the shrub layer, and so thickets of understorey vegetation e.g. Eucalyptus citroidora and Casurina stritica were important habitat elements for many bird species e.g. house sparrow, cattle egret.
\end{abstract}

Keywords: Biotic Relationship, Birds, Damietta Coastal Area, Plants

\section{Introduction}

Avifauna is an important component of Egypt's biological resources. It is the most diverse and prominent of all the country's non aquatic vertebrate fauna. More than 470 bird species are known from Egypt [1]. Egypt enjoys a considerable diversity of habitat, despite its predominantly hyper-arid environment. Lying at the junction of four biogeographically regions, Saharo-sindian, Irano-turanian, Mediterranean and Afrotropical. Egypt has a unique mixture of vegetation types, which support a corresponding diversity of faunal elements [2].

Before construction a facility, a vegetation survey is valuable to document species composition, abundance, and usefulness to birds. Vegetation survey was conducted at the site to 1 ) 
characterize the abundance and structure of vegetation; 2) identify plants with utility as food, nesting material, or tools; 3) determine the abundance and distribution of useful vegetation; and 4) identify and possibly hazardous vegetation. The first major work on the ornithology of Egypt since Shelly [3] was that of Meinertzhagen [4] which included considerable information not only on the distribution of birds in Egypt, but also on their habitats. Another work in Egyptian avian fauna was at the book "birds known to occur in Egypt" by Tharwat [5]. Publications dealing with the ornithology of the Red Sea and Eastern Desert [6,7].

Survey was done at Damietta governorate by Support for Environmental Assessment and Management (SEAM) [8]; this study recorded 32 resident breeding birds inhabiting the governorate. Recently, the first scientific study has been taken at Damietta Governorate to survey all resident, migratory and passage birds by ElArabany et al. [9], they reported 26 resident breeding birds, 15 species passage migrants, and 25 species winter visitors were censused.

When examining avian populations, it is often helpful to relate bird measures to vegetation characteristics of the different habitats. MacArthur\& MkcArthur [10] found that bird species diversity was positively related to vegetation diversity. In general, vegetation vertical diversity, canopy height and cover, and/or total vegetation volume can help explain avian richness and diversity [11,12].

The coastal area of Damietta is exceptionally important for the habitats and the ecosystems that they encompass and for the survival of species dependent upon them. The protection of such area is one way of approaching biodiversity conservation. The proper utilization of the coasts plays a define roles in the development of the region so countries of arid and semi-arid regions direct their efforts towards the renewable resources of coasts ecosystem to produce more food for people and animals, such efforts should be based on previous knowledge of climate, site, vegetation and wild life as well as human activities [13-15].

The present study aimed to evaluate the biotic relationships between growing plants and common birds at different habitat types in the coastal area of Damietta. The obtained results will be useful for credible conservation and management of biodiversity of the study area.

\section{Study Area}

This study was conducted in the coastal area of Damietta. This region extends from Port-Said at east to Dakahleya at west by coast of about 61 $\mathrm{Km}$. It is characterized by villages and summer resorts such as Ezbit El-Burg, Gamasah, New Damietta and Ras El-Bar [14]. The study area was divided into three sites as follow: Site I was located at the western section of Damietta coastal area (El-Kasara), Site $\Pi$ was located between east of new Damietta up to west of Damietta harbour sea-port where interference by man is substantial and Site $\amalg$ was located in the eastern section of the study area at triangle zone of Manzala Lake (Fig. 1).

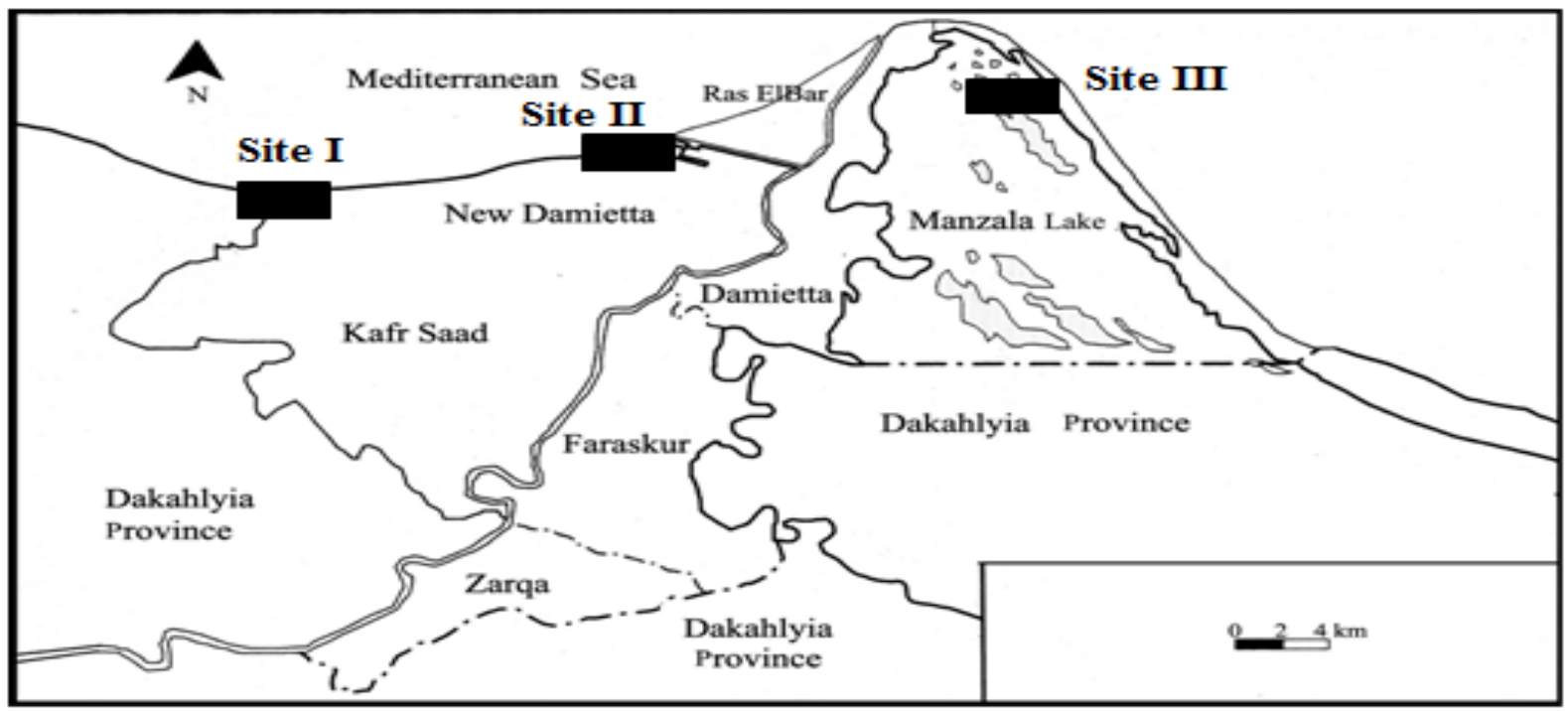

Fig. 1 Location map showing the three sites of the Damietta coastal area. (I) Western section of New Damietta, (П) Distance between east New Damietta up to west of Damietta Harbor, and (Ш) Triangle zone of Lake Manzala (wetland). 


\section{Materials and methods}

\section{Plant Survey}

Fourty stands were selected to represent variation in the habitats. These habitats were sandy habitat (sheets \& dunes) and salt marsh. Locations of the stands were selected after the primary reconnaissance of the area. The distribution of these stands was 20 stands of sand habitat and 20 stands of the salt marsh. The number and distribution of wild plants in each habitats type depended mainly on the presence of a reasonable degree of visual physiographic and physiognomic homogeneity and when a minimum degree of disturbance was ensured. List of plant species, their occurrence are expressed based on frequency, density and dominance. The combined estimation of cover abundance scale is used together with sociability value Braun-Blanquet [16]. Species richness of each species is calculated as the mean number of species per stand according to Pielou [17]. Nomenclature and identification of the plant species followed Täckholm [18]; Boulous [19].

\section{Bird Survey}

Surveys of 20 minutes were conducted from sunrise until 4 hours after sunrise. Each location was visited two times monthly. Over a period of 6 minutes, we slowly walked the $100-\mathrm{m}$ transect, recording each bird seen and heard within 30 minutes on either side of the transect line. At the end of the transect, we stopped and recorded birds for an additional 8 min, then walked back along the transect for 6 min to the starting point [20]. Birds were identified according to Tharwat [5].

\section{Biotic relation between plants and birds}

Biotic relation between plants and birds based on field observations e.g. bird activities such as tracks, burrowing, feeding, nesting and defection were made regularly on the study area.

\section{Results}

\section{Plant Survey}

Field study showed 101 species of plants were recorded. The recorded species were belonged to
49 families, of these 51 species were wild and 43 species were cultivated (Table 1 and Fig. 2).

The most common plants are namely Zygophyllum aegyptium, Phragmites australis, Bassia indica, Halocnemum strobilaceum and Arthrocnemum macrostachyum, Oryza sativa, Triticum aestivum, Phoenix dactylifera, Shinus terebinthifolius, Schinus molle, Zea mays l., Lycopersicon esculentum and Tamarix nilotica.

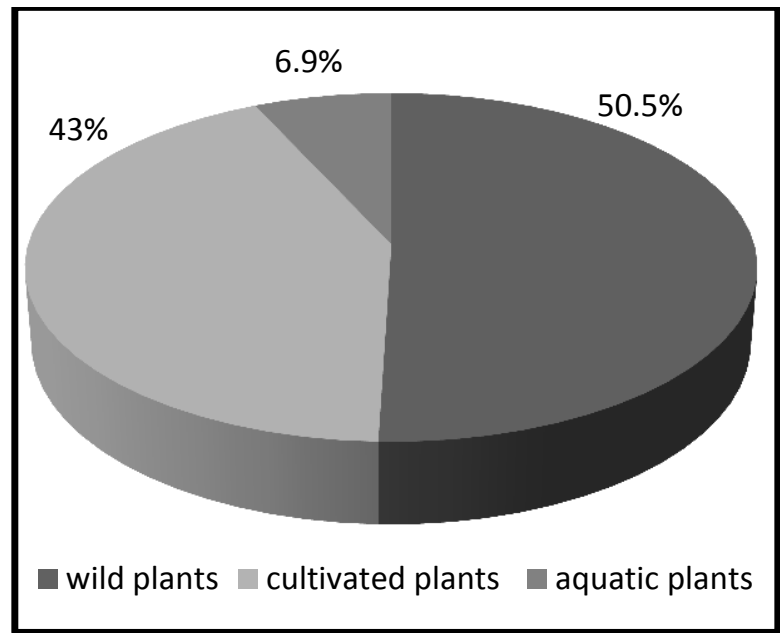

Fig. 2 The percent between wild, cultivated and aquatic plants in the coastal area of Damietta (September, 2009 to March, 2011).

\section{Bird Survey}

The avian community of Damietta coastal area may be classified into four types according to type of feeding: twenty of carnivores species, twenty one of insectivorous species, twenty one of omnivores species and four species of herbivores in a percent of $30.30 \%, 31.82 \%$, $31.82 \%$ and $6.06 \%$ respectively (Fig. 3 ).

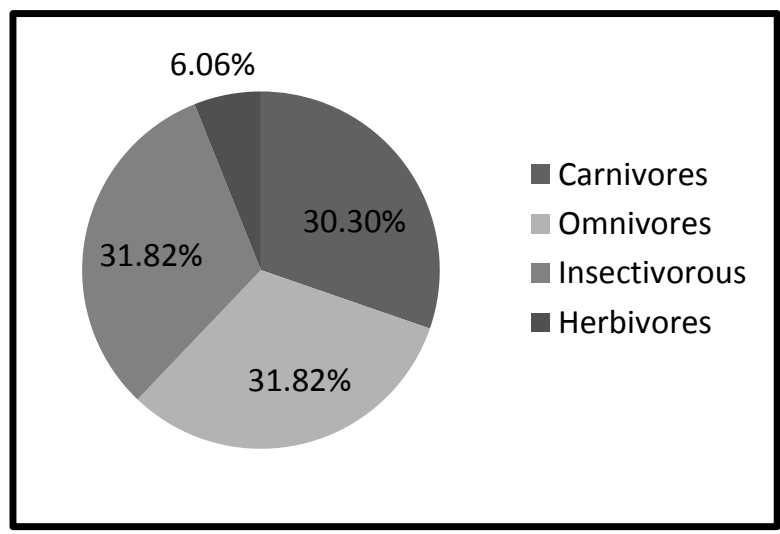

Figure 3: The percent between carnivores, insectivorous, omnivores and herbivores of 66 bird species censused in Damietta coastal area (September, 2009 to March, 2011). 
Table 1 List of plant species recorded in the different habitats of the study area. SB: Sand Bar, SM: Salt March, H: Hummock, DSM: Dry Salt March, WSM: Wet Salt March, RS: Reed Swamp, FF: Fish Farm, FCu: Fertile Cultivated land, WsL: Waste Land, AQ: Aquatic Habitats, G: Grandness, D: dominant, A: Abundant, F: Frequent, O: Occasional, R: Rare ( >5).

a) Terrestrial plants

1. Wild plants recorded in the different habitats of the study area

\begin{tabular}{|c|c|c|c|c|c|c|c|c|c|c|c|}
\hline Family & Scientific name & Arabic name & Use and value & SB & $\mathrm{H}$ & $\begin{array}{c}\text { DS } \\
\text { M }\end{array}$ & $\begin{array}{c}\text { WS } \\
\text { M }\end{array}$ & $\mathrm{RS}$ & $\mathrm{FF}$ & WsL & $\mathrm{G}$ \\
\hline Aizoaceae & Mesembryanthemum nodiflorum & الغاسول الرفيع & Medicinal & $\mathrm{A}$ & $\mathrm{D}$ & & & & & & \\
\hline Aizoaceae & Mesembryanthemum crystallinum & الغاسول / الثلج & Medicinal & $\mathrm{A}$ & $\mathrm{D}$ & & & & & & \\
\hline Amaranthaceae & Amaranthus ascendens & عرف الديك & Weed & & & & & & & A & \\
\hline Apiaceae & Ammi majus & الخلة & Medicinal & & & & & & & & \\
\hline Asclepidia & Cynanchum acutum & المديد & Medicinal, Wood & & & $\mathrm{A}$ & & A & & & \\
\hline Asteraceae & Inula crithmoides & الانيو لا & Medicinal & & & $\mathrm{D}$ & $\mathrm{D}$ & & & & \\
\hline Asteraceae & Sonchus oleraceus & الجعضيض & Edible & & & & & & & $\mathrm{A}$ & \\
\hline Asteraceae & Cichorium pumilum & السريس & Medicinal, weed & & & & & & & & \\
\hline Asteraceae & Pluchea dioscoridis & البرنوف & & & & & & & & & \\
\hline Asteraceae & Urospermum picroides & السليس & Medicinal & & & & & & & $\mathrm{A}$ & \\
\hline Asteraceae & Senecio desfontainii & المرار & Medicinal & $\mathrm{A}$ & $\mathrm{A}$ & & & & & & \\
\hline Asteraceae & Aster squamatus & الاستر & Weed & & & & & & & $\mathrm{A}$ & \\
\hline Brassicaceae & Sisymbrium irio & فجل الجمل & Weed & & & & & & & & \\
\hline Brassicaceae & Cakile maritima & صاروخ البحر & Weed & $\mathrm{D}$ & $\mathrm{D}$ & & & & & & \\
\hline Brassicaceae & Lepidium sativum & حب الرشاد & Medicinal & & & & & & & & $\mathrm{A}$ \\
\hline Caryophyllaceae & Spergularia marina & أبو غلام & Weed & & & $\mathrm{A}$ & & & & & \\
\hline Chenopodiaceae & Halocnemum strobilaceum & الخريزة & Medicinal, halophytes & & $\mathrm{D}$ & $\mathrm{D}$ & & & & & \\
\hline Chenopodiaceae & Beta vulgaris & السلق & Edible & & & & & & & & \\
\hline Chenopodiaceae & Salsola kali & السالسو لا & Medicinal & $\mathrm{A}$ & $\mathrm{A}$ & & & & & & \\
\hline Chenopodiaceae & Chenopodium murale & الزربيح & Medicinal & & & & & & & A & \\
\hline Chenopodiaceae & Bassia indica & الكوخيا & Feeder for animals & & & $\mathrm{A}$ & & & A & & \\
\hline Convolvolaceae & Convolvulus arevensis & العليق & Medicinal, Wood & & & & & & & $\mathrm{O}$ & \\
\hline Cyperaceae & Cyperus rotundus & السعد & Medicinal & & & & & & & & \\
\hline Euphorbiaceae & Euphorbia peplus & اللبينة & Medicinal & & & & & & & & $\mathrm{D}$ \\
\hline Fabaceae & Alhagi graecorum & العاقول & Medicinal, halophytes & $\mathrm{D}$ & $\mathrm{D}$ & & & & & & \\
\hline Fabaceae & Lotus glaber & رجل العصفور & Weed & & & & & & & & $\mathrm{D}$ \\
\hline Fabaceae & Medicago sativa & النفل الن & Weed & & & & & & & & $\mathrm{D}$ \\
\hline Fabaceae & Melilotus indicus & الحندقوق & Weed & & & & & & & & \\
\hline Juncaceae & Juncus acutus & السمار الحلو & Industry cages, mats & & & & A & & & & \\
\hline Labiatae & Mentha microphylla & حبق البحر & Seeds are eaten by birds & & & & & & & $\mathrm{O}$ & \\
\hline Malvaceae & Malva parviflora & الخبيزة & Edible & & & & & & & $\mathrm{D}$ & \\
\hline
\end{tabular}


Table 1 continued

\begin{tabular}{|l|}
\hline Onagraceae \\
\hline Plantagonaceae \\
\hline
\end{tabular}

Poaceae

Poaceae

Poaceae

Poaceae

Poaceae

Poaceae

Polygonaceae

Polygonaceae

Polygonaceae

Portulaceae

Primulaceae

Solanaceae

Solanaceae

Typhaceae

Uriticaceae

Verbenaceae

Zygophyllaceae

Tamarixaceae

Ludwigia stolonifera

Plantago major

Imperata lastical

Arundo donax

Lolium temulentum

Polypogon monspleinsis

Setaria viridis

Avena fatua

Persicaria salicifolia

Polygonum equisetiforme

Rumex dentatus

Portulaca oleracea

Anagallis arvensis

Solanum nigrum

Datura stramonium

Typha domingensis

Uritica urens

Phylla nadiflora

Zygophyllum album

Tamarix nilotica

\begin{tabular}{|c|c|c|}
\hline$r a$ & جوسيا & Weed \\
\hline & لسان الحمل & Medicinal \\
\hline & الحلفا & Paper production \\
\hline & الغاب البلدي & Industry cages \\
\hline & دهتورة & Medicinal \\
\hline einsis & ديل القط & Weed \\
\hline & ديل الفار & Weed \\
\hline & الزمبر & Feeder for livestock \\
\hline$\overline{l i a}$ & أبو زلف & Seeds are eaten by birds \\
\hline iforme & ق قرضاب & Weed \\
\hline & الحميض & Food, Edible \\
\hline & الرجلة & Medicinal, Edible \\
\hline & عين القط & Weed \\
\hline & عنب الديب & Medicinal \\
\hline$l$ & الداتور اه & Medicinal \\
\hline & البردي & Mats \\
\hline & 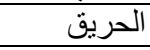 & Medicinal \\
\hline & اللبييا & Medicinal \\
\hline$\imath$ & الرطريط & Medicinal, halophyte \\
\hline & الطرفة & Ornamental \\
\hline
\end{tabular}

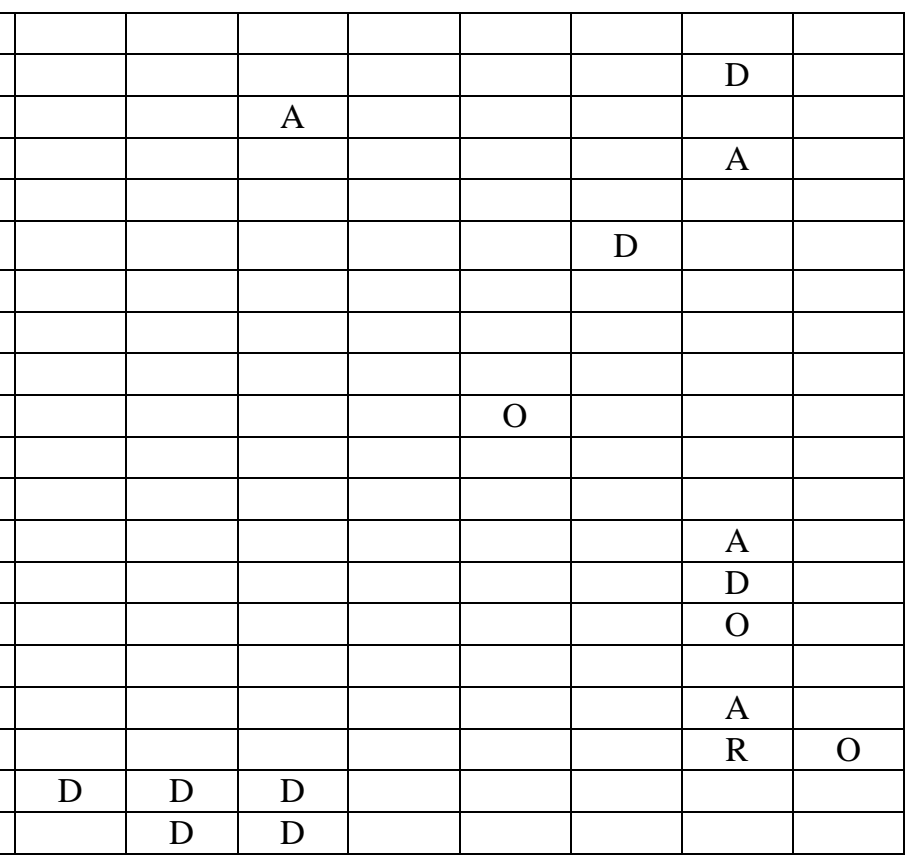

2. Cultivated plants recorded in the different habitats of the study area

\begin{tabular}{|c|c|c|c|c|c|c|c|c|c|c|c|}
\hline Family & Scientific name & $\begin{array}{c}\text { Arabic } \\
\text { name }\end{array}$ & Use and value & $\boldsymbol{H}$ & DSM & WSM & $R S$ & $F F$ & $F C u$ & WsL & $G$ \\
\hline Anacardiaceae & Shinus terebinthifolius & الفلفل العريض & Ornamental, hedge, Medicinal & & & & & & $\mathrm{D}$ & & $\mathrm{D}$ \\
\hline Anacardiaceae & Schinus molle & الفلفل الرفيع & Ornamental, hedge & & & & & & $\mathrm{F}$ & & $\mathrm{F}$ \\
\hline Anacardiaceae & Mangifera indica & مانجو & Food & & & & & & & & $\mathrm{O}$ \\
\hline Apiaceae & Apium graveolens & الكرفس & Medicinal & & & & & & $\mathrm{A}$ & & $\mathrm{A}$ \\
\hline Apiceae & Anethum graveolens & الشبت & Medicinal & & & & & & & & \\
\hline Apocynaceae & Thevetia peruviana & الثيفيتا & Ornamental, poisonous & & & & & & & & $\mathrm{A}$ \\
\hline Asteraceae & Helianthus annus & عباد الشمس & Oil, Food & & & & & & & & \\
\hline Bignoniaceae & Jacaranda acutifolia & الجاكار اندا & Medicinal & & & & & & & & $\mathrm{R}$ \\
\hline Brassicaceae & Brassica oleraceae & الكرنب & Food & & & & & & & & \\
\hline Casuarinaceae & Casuarina stricta & الكازورينا & Wind break, wood & & & & & & $\mathrm{D}$ & & \\
\hline Cesalpiniaceae & Delonix regia & البو انسيانا & Ornamental & & & & & & & & $\mathrm{A}$ \\
\hline Cucurbitaceae & Cucurbita pepo & كوسة & Food & & & & & & A & & \\
\hline Cupressaceae & Cupressus semperviren & | - السرو & Ornamental, Wood & & & & & & & & $\mathrm{O}$ \\
\hline
\end{tabular}




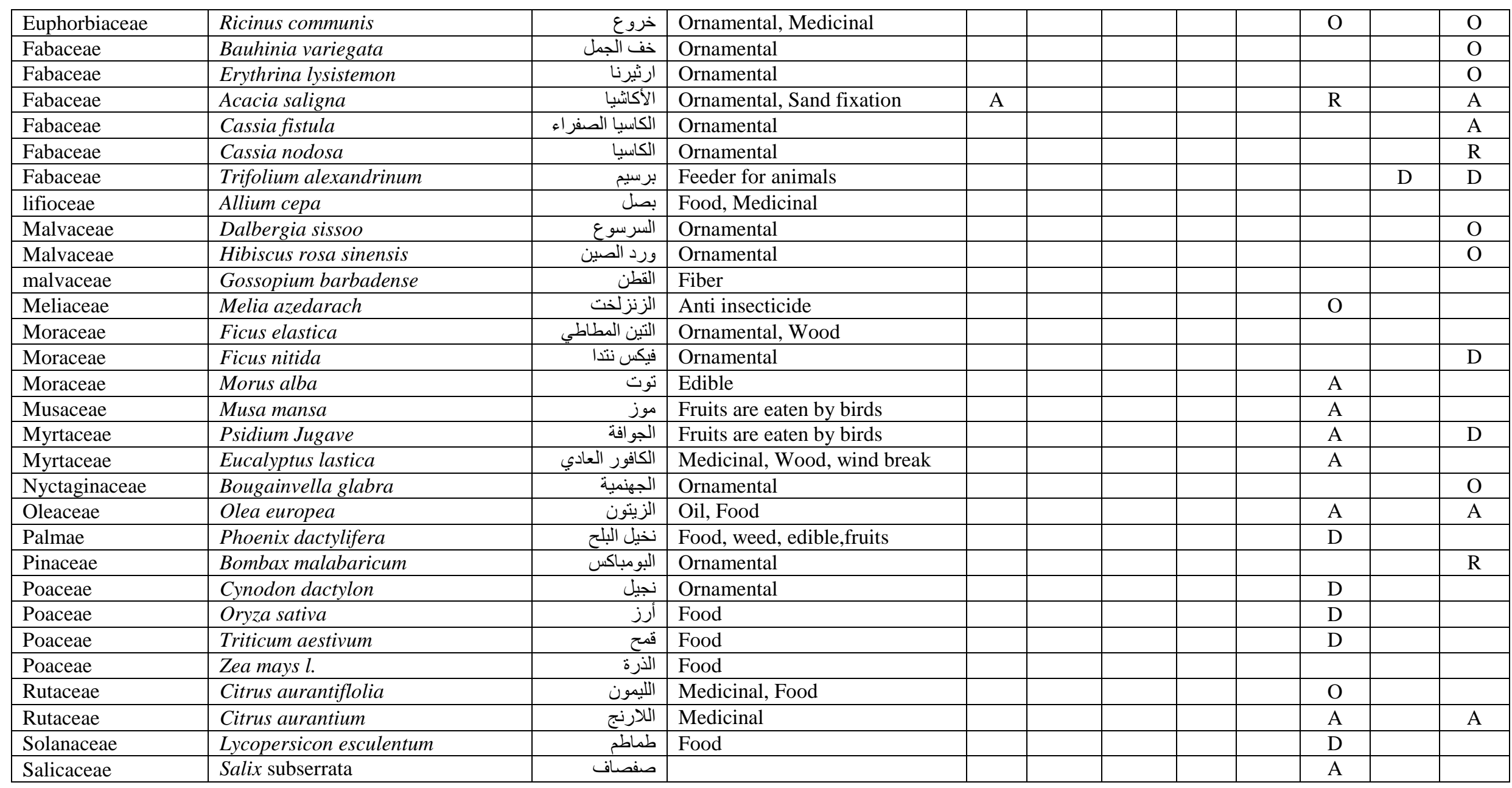

\section{b) Aquatic Plants}

\begin{tabular}{|c|c|c|c|c|c|c|c|c|c|c|c|}
\hline Family & Scientific name & Arabic name & Use and value & SB & $\mathrm{H}$ & $\begin{array}{c}\text { DS } \\
\text { M }\end{array}$ & $\begin{array}{l}\text { WS } \\
\text { M }\end{array}$ & $\mathrm{RS}$ & FF & WsL & AQ \\
\hline Araceae & Pistia stratiotes & الزقيم & Free floating & & & & & & & & $\mathrm{D}$ \\
\hline Araceae & Lemna gibba & عدس الماء & Duckweed & & & & & & & & $\mathrm{D}$ \\
\hline Onagraceae & Ludwigia stolonifera & جوسيا & & & & & & & & & $\mathrm{A}$ \\
\hline Poaceae & Phragmites australis & البوص & Sand fixation, water treatment & $\mathrm{F}$ & $\mathrm{D}$ & $\mathrm{F}$ & $\mathrm{A}$ & $\mathrm{D}$ & $\mathrm{D}$ & $\mathrm{D}$ & $\mathrm{D}$ \\
\hline Poaceae & Echinochloa stagnina & أمشوط & Feeder, forage & & & & & $\mathrm{D}$ & & & $\mathrm{D}$ \\
\hline
\end{tabular}




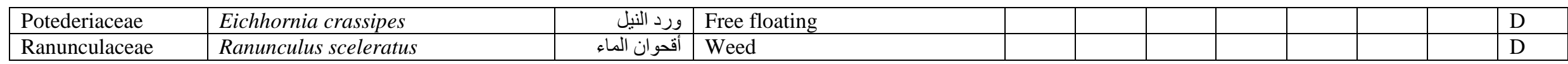

Table 2 Survey, classifications, feeding of birds distributed in different habitats in the coastal area of Damietta at August, 2009 to February, 2011. M: Marine habitat, Co: Coastal habitat, W: Wetland habitat, Cu: Cultivated land, U: Urban habitat, C: Common, A: Abundant, R: Rare, SC: Scare

\begin{tabular}{|c|c|c|c|c|c|c|c|c|c|c|}
\hline \multirow{2}{*}{ Order } & \multirow{2}{*}{ Family } & \multirow{2}{*}{ Scientific name } & \multirow{2}{*}{ Common name } & \multirow{2}{*}{ الاسم باللغة العربية } & \multirow{2}{*}{ Feeding } & \multicolumn{5}{|c|}{ Habitats } \\
\hline & & & & & & $\mathrm{Ma}$ & $\mathrm{Co}$ & $\mathrm{W}$ & $\mathrm{Cu}$ & $\mathrm{U}$ \\
\hline \multirow[t]{4}{*}{ Ciconiiformes } & \multirow[t]{4}{*}{ Ardidae } & Egretta garzetta & Little egret & البلثون الأبيض & Carnivores & & & $\mathrm{A}$ & $\mathrm{R}$ & \\
\hline & & Ardeolar alloides & Squacco heron & الواق الأبيض & Carnivores & & & $\mathrm{R}$ & $\mathrm{R}$ & \\
\hline & & Bubulcus ibis & Cattle egret & أبو قردان & Carnivores & & & $\mathrm{R}$ & A & $\mathrm{R}$ \\
\hline & & Ardea cinerea & Grey heron & البلشون الرمادي & Carnivores & & & SC & & \\
\hline \multirow[t]{4}{*}{ Columbiformes } & \multirow[t]{4}{*}{ Columbidae } & Columba livia & Rock dove & الحمام الجبلي & Herbivores & & $\mathrm{R}$ & $\mathrm{R}$ & $\mathrm{R}$ & $\mathrm{R}$ \\
\hline & & Columba l. domestica & Feral pigeon & الحمام المنزلي & Herbivores & & $\mathrm{A}$ & $\mathrm{A}$ & $\mathrm{A}$ & $\mathrm{A}$ \\
\hline & & Streptopelia decaocto & Collared dove & اليمام المطوق & Herbivores & & & $\mathrm{A}$ & $\mathrm{A}$ & $\mathrm{A}$ \\
\hline & & Columba aegyptiaca & Palm Dove & اليمام البلدي & Herbivores & & $\mathrm{A}$ & $\mathrm{A}$ & $\mathrm{A}$ & $\mathrm{A}$ \\
\hline \multirow[t]{5}{*}{ Gruiformes } & \multirow[t]{5}{*}{ Rallidae } & Rallus aquaticus & Water rail & مرعة الماء & Omnivores & & & $\mathrm{R}$ & $\mathrm{R}$ & \\
\hline & & Gallinula chloropus & Moorhen & دجاج الماء & Omnivores & & & $\mathrm{R}$ & $\mathrm{R}$ & \\
\hline & & Crexcrex & Corncrake & مرعة الغلة & Omnivores & & & $\mathrm{SC}$ & & \\
\hline & & Arenaria interpret & Turnstone & قنبرة الماء & Omnivores & & $\mathrm{C}$ & $\mathrm{C}$ & $\mathrm{C}$ & \\
\hline & & Fulica atra & Coot & الغر & Omnivores & & & $\mathrm{C}$ & & \\
\hline \multirow[t]{2}{*}{ Acciptiformes } & \multirow[t]{2}{*}{ Acciptridae } & Elanus caeruleus & Black-winged kite & الحدأة ذات الجناح الأسود & Carnivores & & & & $\mathrm{R}$ & \\
\hline & & Cicusae ruginosus & Marsh harrier & الدراع & Carnivores & & $\mathrm{SC}$ & & & \\
\hline Falconiformes & Falconidae & Falco tinnunculus & Kestrel & العوسق & Carnivores & & $\mathrm{R}$ & $\mathrm{R}$ & $\mathrm{R}$ & $\mathrm{R}$ \\
\hline Cuculiformes & Cuculidae & Cuculus aegypticus & Sengal Coucal & الكوكو & Insectivorous & & & $\mathrm{SC}$ & $\mathrm{SC}$ & \\
\hline Strigiformes & Strigidae & Athene noctua & Little Owl & أم قويق & Carnivores & & & & $\mathrm{R}$ & $\mathrm{R}$ \\
\hline Galliformes & Phasianidae & Coturnix coturnix & Migratory quail & 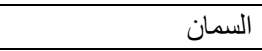 & Omnivores & & $\mathrm{C}$ & & & \\
\hline Caprimulgiformes & Caprimulgidae & Caprimulgus europaeus & Nightjar & البخاخ & Insectivorous & & $\mathrm{SC}$ & & & \\
\hline \multirow[t]{10}{*}{ Passeriformes } & Corvidae & Corvus corone cornix & Hooded crow & الغراب البلدي & Omnivores & & $\mathrm{C}$ & $\mathrm{C}$ & $\mathrm{C}$ & $\mathrm{C}$ \\
\hline & Alaudidae & Galerida cristata & Crested lark & قنبرة متوجة & Omnivores & & & $\mathrm{R}$ & $\mathrm{R}$ & \\
\hline & Passeridae & Passer domesticus & House sparrow & العصفور الدوري & Omnivores & & & $\mathrm{C}$ & $\mathrm{C}$ & $\mathrm{C}$ \\
\hline & \multirow[t]{4}{*}{ Hirundinidae } & Hirundo $r$. savigmii & Egyptian barn swallow & عصفور الجنة المصري & Insectivorous & & $\mathrm{C}$ & $\mathrm{C}$ & $\mathrm{C}$ & $\mathrm{C}$ \\
\hline & & Riparia riparia & Sand martin & سنونو & Insectivorous & & $\mathrm{R}$ & $\mathrm{R}$ & $\mathrm{R}$ & \\
\hline & & Lanius excubitor & Great grey shrike & دقناش البادية & Carnivores & & & & $\mathrm{SC}$ & \\
\hline & & Hirundo rustica rustica & European barn swallow & عصفور الجنة الأوروبي & Insectivorous & & $\mathrm{R}$ & & & $\mathrm{R}$ \\
\hline & Pycnonotidae & Pycnonotus barbatus & Common bulbul & بلبل شاع & Omnivores & & & $\mathrm{SC}$ & $\mathrm{SC}$ & $\mathrm{SC}$ \\
\hline & Motacillidae & Anthusno vaeseelandiae & Richard's pipit & أبو فصية & Insectivorous & & $\mathrm{SC}$ & & & \\
\hline & Laniidae & Lanius collorio & Red-backed shrike & دقناش أكحل & Carnivores & & $\mathrm{SC}$ & & & \\
\hline
\end{tabular}




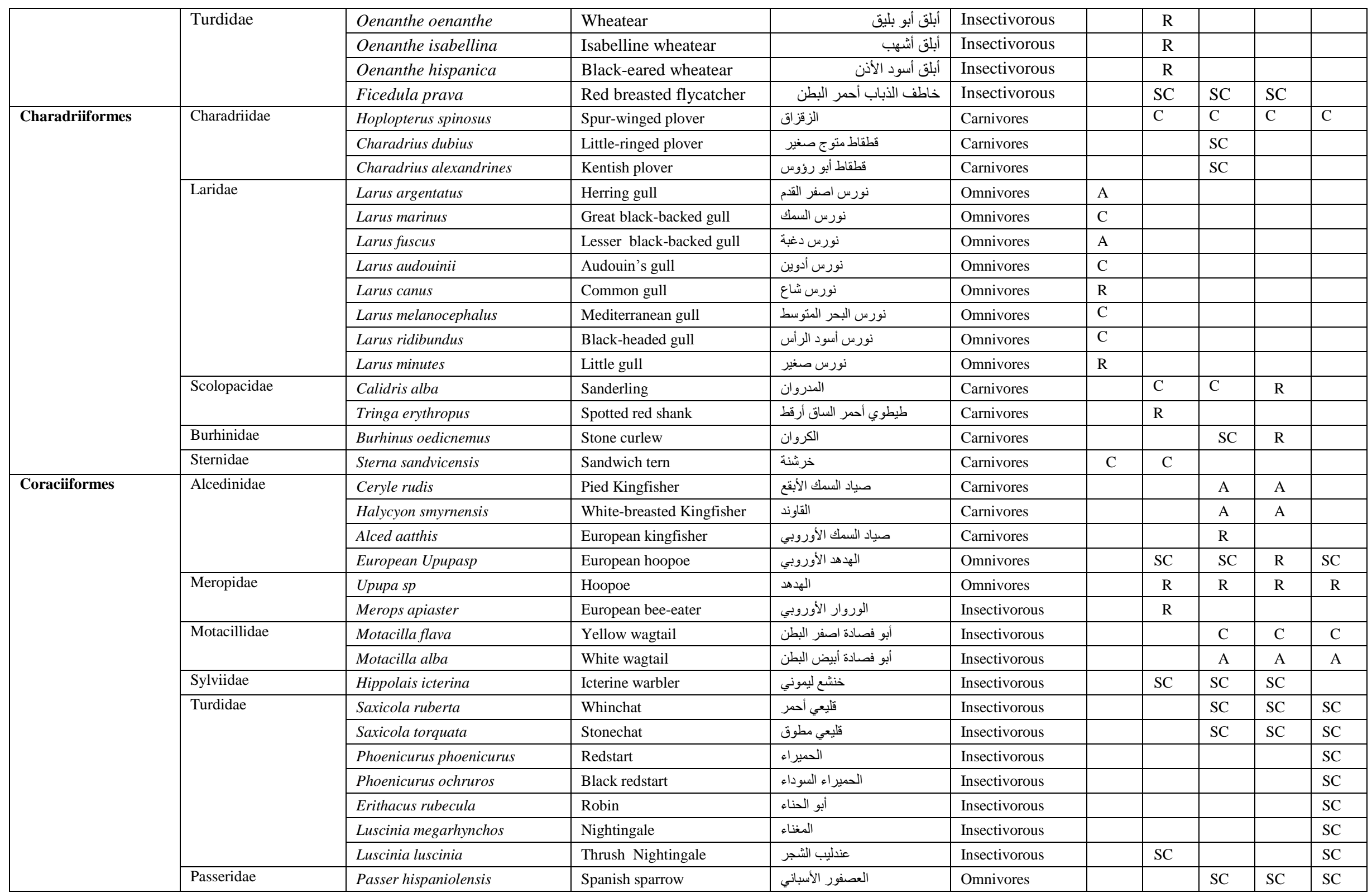


Bird survey of selected sites were 68 birds species, they were belonged to 12 orders and 28 families. These species and their classification and distribution in different habitats were listed in Table (2) and figure (4).

Field study indicated that the major habitat types were recognized in the study area could be divided into aquatic and terrestrial habitats. The aquatic habitats were namely: irrigation canals, drains, ditches and fish farms, however the terrestrial habitats are beach zone, sand bar, mounds, hummocks, hillocks, sand flats, salt marshes (wet \& dry), reed swamps, fertile cultivated lands, highway road side and reclaimed lands.

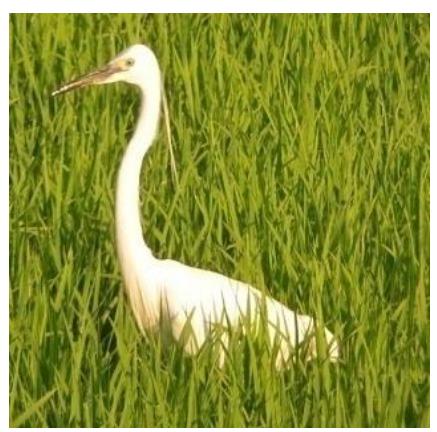

Little egret

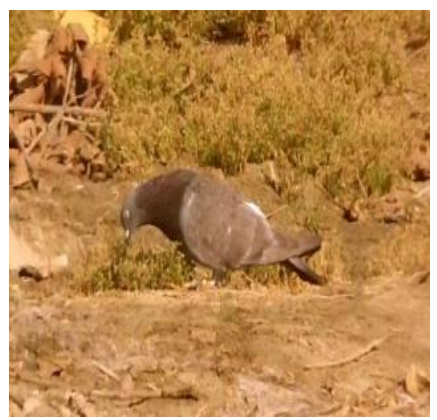

Rock dove

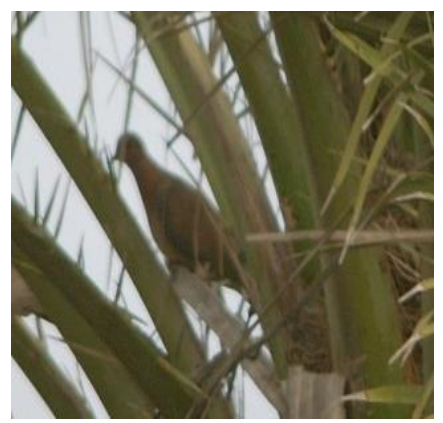

Palm Dove

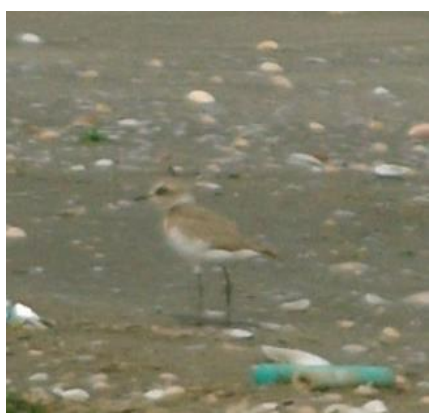

Turnstone

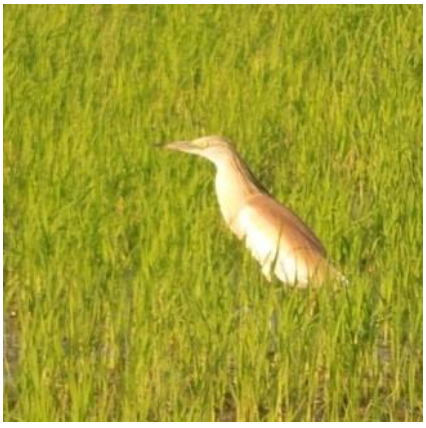

Squacco heron

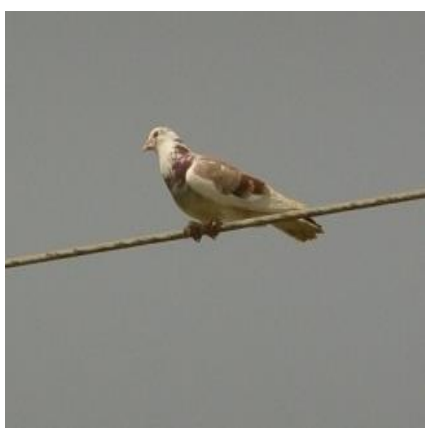

Feral pigeon

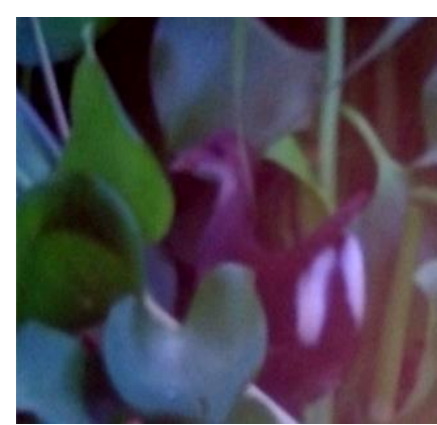

Water rail

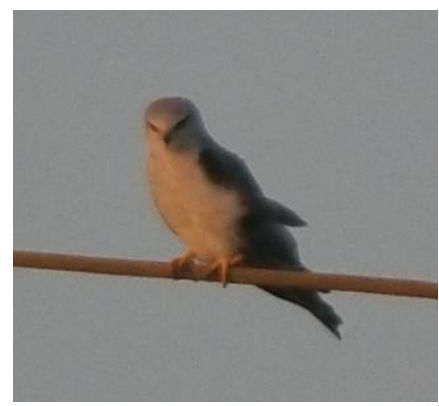

Black-winged kite

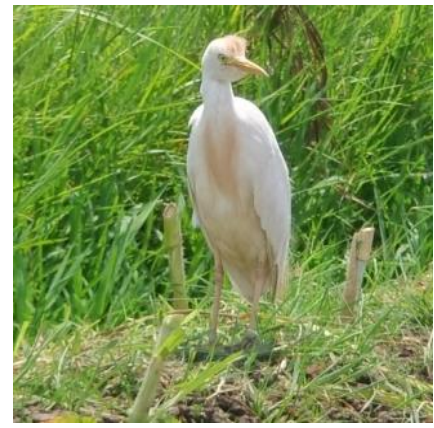

Cattle egret

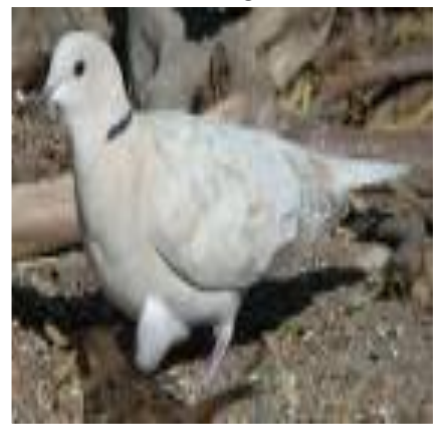

Collared dove

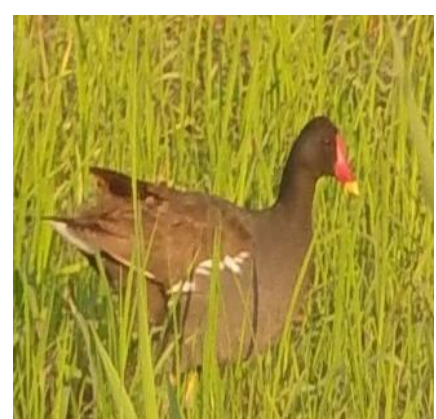

Moorhen

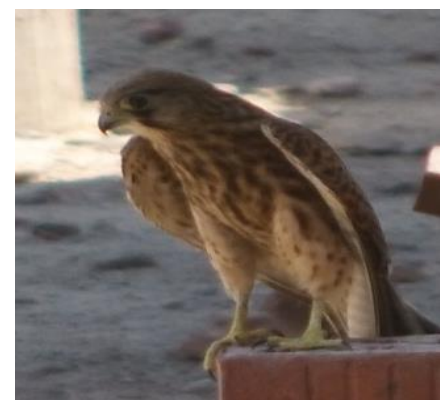

Kestrel

Fig. 4 The most common birds observed during the field study in the coastal area of Damietta. Photos by Habib, S.G. 


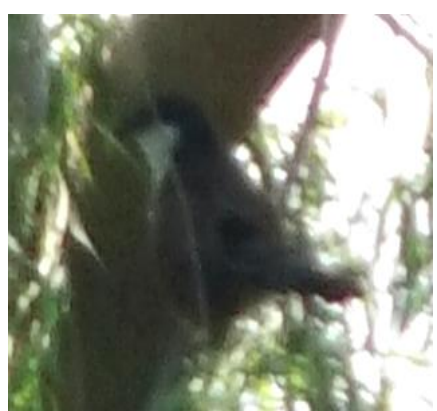

Senegal Coucal

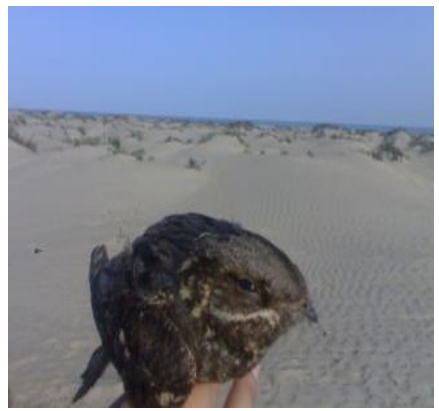

Nightjar

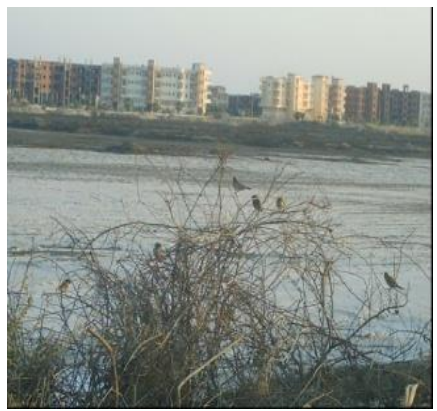

House sparrow

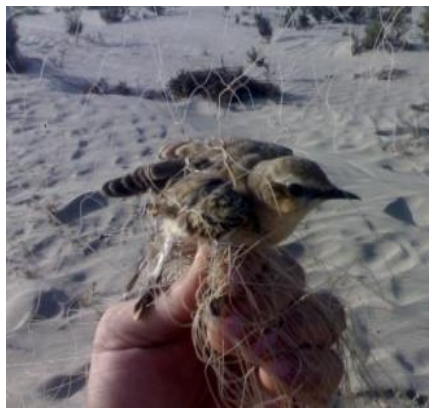

Isabelline wheatear

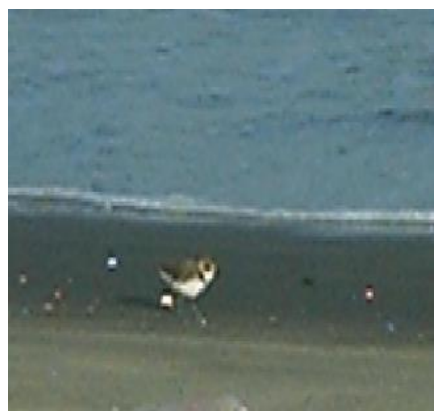

Kentish plover

Fig. 4 Continued

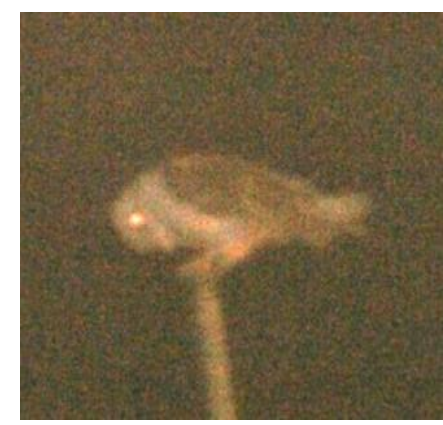

Little Owl

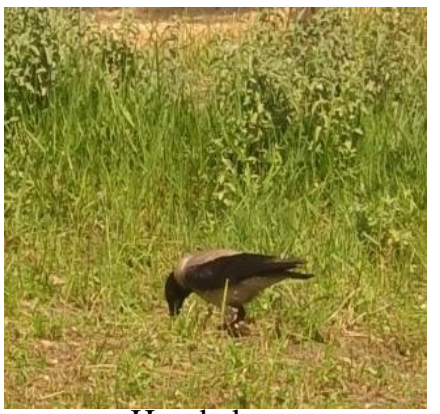

Hooded crow

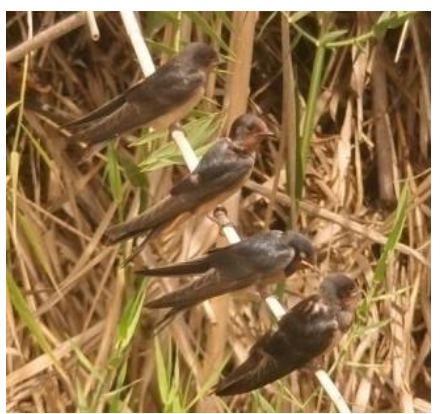

Egyptian barn swallow

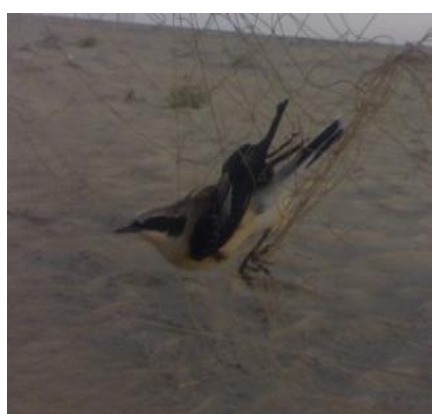

Black-eared wheatear

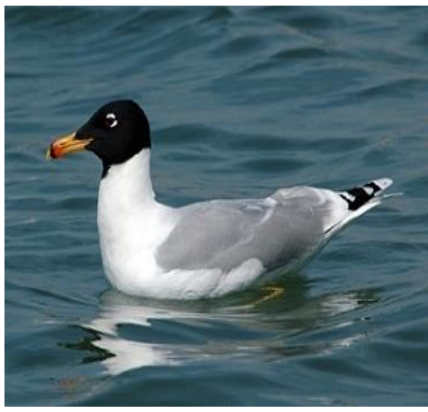

Great black-backed gull

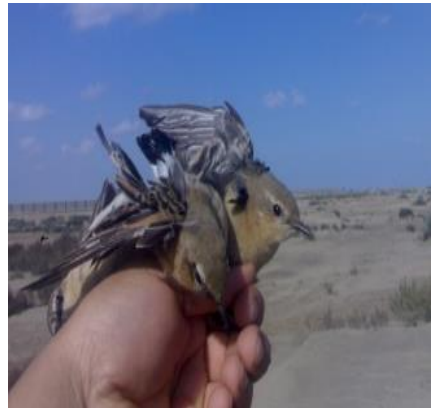

Migratory quail

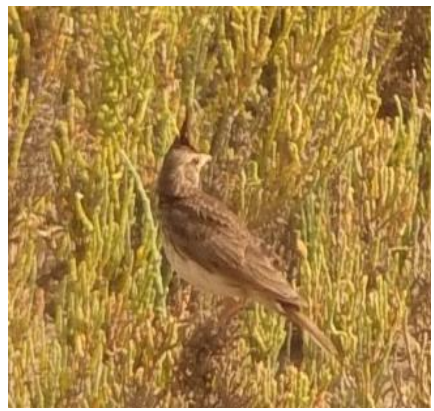

Crested lark

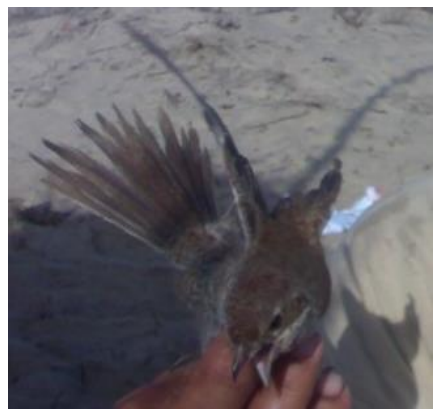

Red-backed shrike

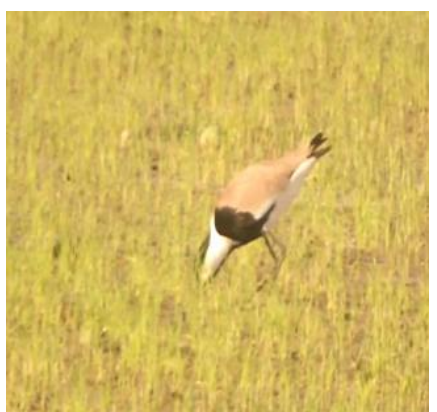

Spur-winged plover

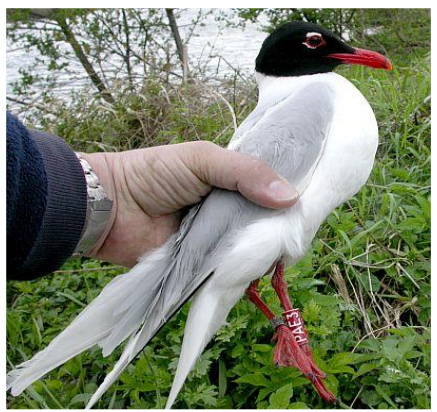

Mediterranean gull 


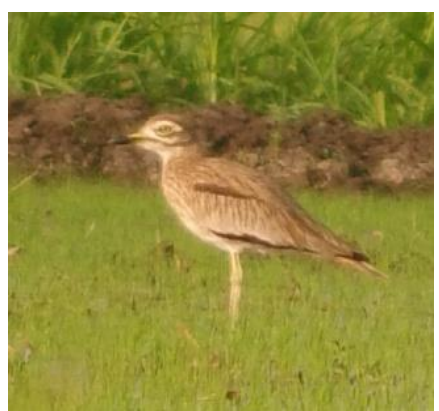

Stone curlew

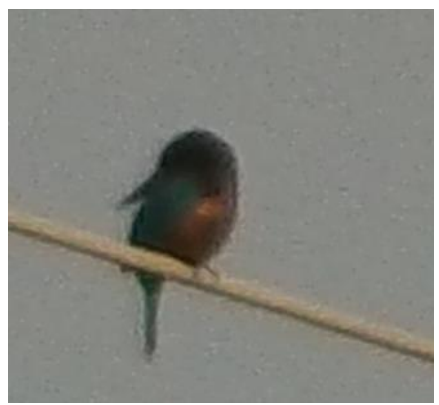

European kingfisher

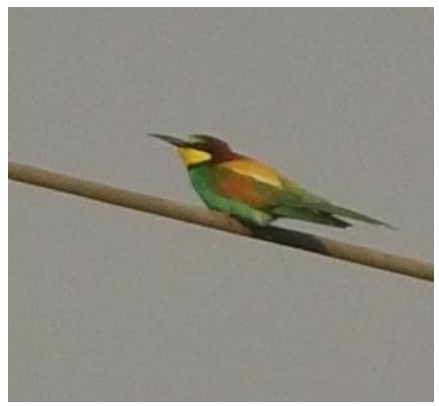

European bee-eater

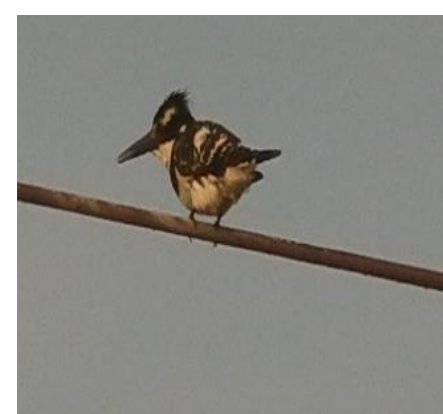

Pied Kingfisher

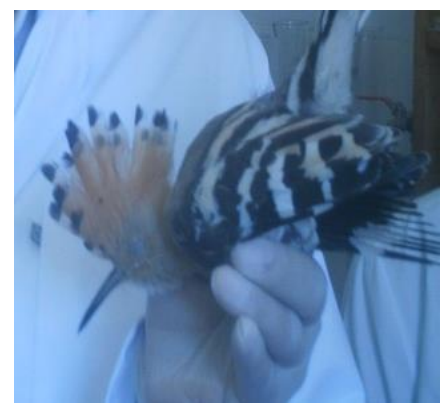

European hoopoe

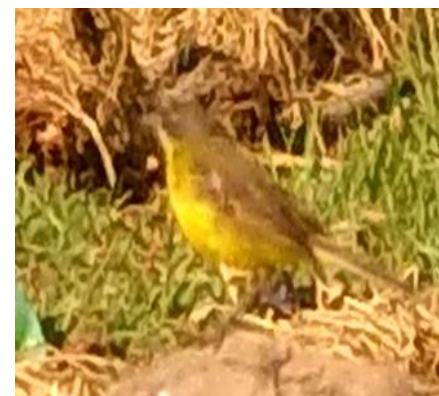

Yellow wagtail

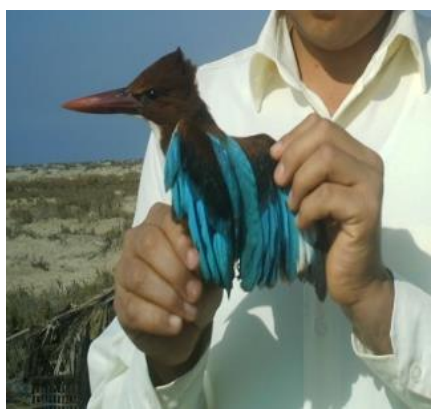

White-breasted Kingfisher

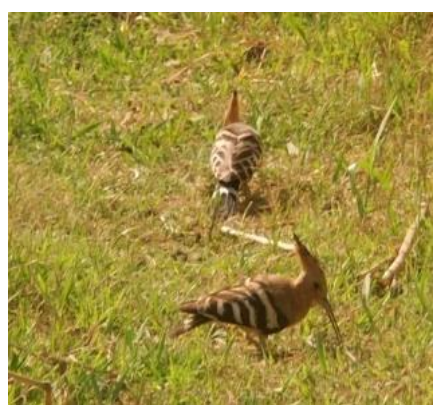

Hoopoe

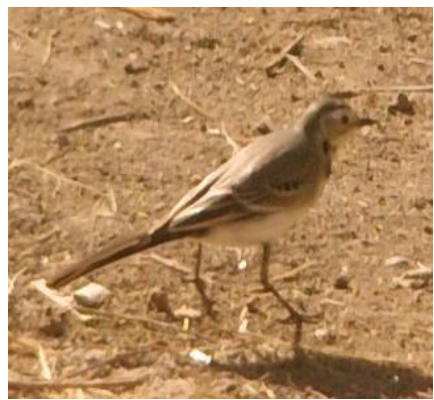

White wagtail

Fig. 4 Continued

\section{Biotic Relations between Plants and Birds}

Based on field observations the relationship between plants and birds was as following:

\section{Feeding}

Plants provide food for birds both directly and indirectly. Directly as birds can eat many parts of plants, including buds, berries, fruit, nuts and seeds. there were 13 bird species that feed directly (Table 3 ) or indirectly as plants provide habitat for insects which inturn are eaten by birds and they were 15 bird species showed in (Table 4).

\section{Shelter and protection}

Field observations showed that plants provided a natural shelter in the study area to attract birds to a safe and secure setting. There were 26 birds used plants as shelter (Table 5).

\section{Nesting material}

Field observation showed that there were three important elements of the design of most nests, including robust, provide good insulation and were well camouflaged. Sticks, bark and grass commonly provide the support structure, and these items were usually abundant. Most eggs that were produced by birds were eaten by predators so there was a high demand for concealed nest sites. Many small birds nest in the understorey where the vegetation was often thickest. Some species also show a preference for nesting in spiky shrubs.

\section{Seed dispersal}

Some species of bird e.g. Common bulbul and Hoopoe are known to include some fruit in their diet. Even primarily insectivorous species such as Egyptian barn swallow, house sparrow will occasionally take small fruits. This is, perhaps, a 
result of the broad individual niches of the species in our relatively depauperate avifauna.

\section{Pollination}

Humming birds adapted by going to a flowers and taking the pollen like bees do with insects. Most bees when go to flower get flower pollen on thier legs, then fly to another flower and drop the pollen there thus, dispersing the pollen by visiting different plants.

Table 3 Bird species that feed directly on plants

\begin{tabular}{|l|l|}
\hline \multicolumn{1}{|c|}{ Bird name } & \multicolumn{1}{|c|}{ Direct feeding } \\
\hline $\begin{array}{l}\text { House } \\
\text { sparrow }\end{array}$ & $\begin{array}{l}\text { Seeds of some grasses and weeds. It } \\
\text { preferred oats and wheat and also } \\
\text { eaten berries and fruits. }\end{array}$ \\
\hline Feral pigeon & Grass seeds and berries. \\
\hline $\begin{array}{l}\text { Collared } \\
\text { dove }\end{array}$ & Grain and shoots. \\
\hline Palm doves & Grass, seeds and grains. \\
\hline Rock dove & $\begin{array}{l}\text { Grains of rice, maize, millet and } \\
\text { other cereals. Banyan figs and other } \\
\text { berry-like fruits. }\end{array}$ \\
\hline Water rail & $\begin{array}{l}\text { Buds, seeds, flowers, shoots and } \\
\text { seeds of water plants, berries and } \\
\text { fruit. }\end{array}$ \\
\hline $\begin{array}{l}\text { Moorhen, } \\
\text { Corncrake }\end{array}$ & $\begin{array}{l}\text { Plant material including grass seed } \\
\text { and cereal. }\end{array}$ \\
\hline Turnstone & Coconut. \\
\hline Crested lark & Seeds of weeds. \\
\hline $\begin{array}{l}\text { Common } \\
\text { bulbul }\end{array}$ & Fruit, nectar \\
\hline Herring gull & $\begin{array}{l}\text { Vegetable matter such as roots, } \\
\text { tubers, seeds, grains, nuts and fruit }\end{array}$ \\
\hline $\begin{array}{l}\text { Lesser black- } \\
\text { back gull }\end{array}$ & $\begin{array}{l}\text { Seeds and berries } \\
\text { berries }\end{array}$ \\
\hline Hoopoe \\
\hline
\end{tabular}

\section{Discussion}

A strong correlation between bird community and habitat diversity indices suggests that birds are dependent on the compositional complexity of trees, shrubs, and herbs. These observations suggest that birds community is significantly associated with plant species diversity, confirming the findings of James \& Wamer [21]. To study the biotic relationships between plants and birds in the different habitats at the coastal area of Damietta, it is necessary first to identify the major type habitats and make a survey of both plants and birds.

In the present work the major habitat types being recognized in the study area and were divided into aquatic and terrestrial habitats. The aquatic habitats were namely: irrigation canals, drains, ditches and fish farms, however the terrestrial habitats were beach zone, sand bar, mounds, hummocks, hillocks, sand flats, salt marshes (wet \& dry), reed swamps, fertile cultivated lands, highway road side and reclaimed lands.

Table 4 Bird species that feed indirectly on plants

\begin{tabular}{|l|l|}
\hline \multicolumn{1}{|c|}{ Bird name } & \multicolumn{1}{c|}{ Indirect feeding } \\
\hline Nightjar & $\begin{array}{l}\text { moths and other large flying } \\
\text { insects }\end{array}$ \\
\hline $\begin{array}{l}\text { Egyptian barn } \\
\text { swallow }\end{array}$ & $\begin{array}{l}\text { flying insects Mongooses and } \\
\text { bugs }\end{array}$ \\
\hline $\begin{array}{l}\text { European barn } \\
\text { swallow }\end{array}$ & flying insects \\
\hline $\begin{array}{l}\text { Isabelline } \\
\text { wheatear }\end{array}$ & flying insects \\
\hline $\begin{array}{l}\text { Black-eared } \\
\text { wheatear }\end{array}$ & flying insects \\
\hline $\begin{array}{l}\text { Red breasted } \\
\text { flycatcher }\end{array}$ & flying insects \\
\hline $\begin{array}{l}\text { European bee- } \\
\text { eater }\end{array}$ & bees, wasps and hornets \\
\hline Icterine warbler & flying insects \\
\hline Whinchat & Terrestrial and flying insects \\
\hline Stonechat & flying insects \\
\hline Redstart & flying insects \\
\hline Black redstart & flies \\
\hline Robin & flying insects \\
\hline Nightingale & flying insects \\
\hline $\begin{array}{l}\text { Thrush } \\
\text { Nightingale }\end{array}$ & flying insects \\
\hline
\end{tabular}

Plants survey indicated that there were one hundred and one plant species, belonging to 49 families, of these 51 species were wild and 43 species were cultivated. On the other hand, sixty six species of birds were censused belonged to 12 orders and 28 families. Changes in plant species composition and structure can negatively affect habitat quality [22] and reduce individual fitness [23]. Fragmentation of natural habitats may lead to the local extinction of some species, particularly those that occur at low densities, due to low recolonization rates, increased predation by natural predators and competitors, and other environmental factors $[22,23]$. The present study showed that plants provide food at different times of the year to birds directly or indirectly. Directly in the form of seeds, fruit, buds, seeds, flowers, shoot, berries and nuts, indirectly as plants provide habitat for insects which in turn are eaten by birds. e.g. Egyptian barn swallow, Red breasted flycatcher, European barn swallow and 
European bee-eater.Plants also provide safe nesting sites and cover that protect birds from inclement weather and predation. The most popular natural shelters in the study area included:

Table 5 Birds that used plants as shelter

\begin{tabular}{|c|c|}
\hline Bird name & Bird nest \\
\hline $\begin{array}{l}\text { Little egret, } \\
\text { Squacco heron, } \\
\text { Cattle egret and } \\
\text { Grey heron }\end{array}$ & $\begin{array}{l}\text { On platforms of sticks in trees } \\
\text { or shrubs. The colonies were } \\
\text { found in woodlands near lakes } \\
\text { or rivers, in swamps, or on } \\
\text { small inland or coastal islands. }\end{array}$ \\
\hline $\begin{array}{l}\text { Collared dove, } \\
\text { Palm Dove }\end{array}$ & trees \\
\hline $\begin{array}{l}\text { Water rail, } \\
\text { Moorhen }\end{array}$ & $\begin{array}{l}\text { building its nest a little above } \\
\text { the water level from whatever } \\
\text { plants(Herbs and jungle) are } \\
\text { available nearby }\end{array}$ \\
\hline $\begin{array}{l}\text { Corncrake and } \\
\text { Coot }\end{array}$ & $\begin{array}{l}\text { in grassland, sometimes in safer } \\
\text { sites along a hedge, or near an } \\
\text { isolated tree or bush, or in } \\
\text { overgrown vegetation. }\end{array}$ \\
\hline $\begin{array}{l}\text { Black-winged } \\
\text { kite }\end{array}$ & a loose platform of twigs \\
\hline Stone curlew & $\begin{array}{l}\text { was off the ground in } \\
\text { vegetation }\end{array}$ \\
\hline Senegal coucal & on the branches of trees \\
\hline Little owl & in holes in trees. \\
\hline Hooded crow & a tall tree \\
\hline House sparrow & $\begin{array}{l}\text { in the open, on the branches of } \\
\text { trees, especially evergreens and } \\
\text { hawthorns }\end{array}$ \\
\hline $\begin{array}{ll}\begin{array}{l}\text { Great } \\
\text { shrike }\end{array} & \text { grey } \\
\end{array}$ & on side branches near the trunk \\
\hline Common bulbul & $\begin{array}{l}\text { inside the leafy foliage of a } \\
\text { small tree or shrub }\end{array}$ \\
\hline $\begin{array}{l}\text { Hoopoe and } \\
\text { European } \\
\text { hoopoe }\end{array}$ & $\begin{array}{l}\text { in a hole in a tree or wall, with a } \\
\text { narrow entrance }\end{array}$ \\
\hline $\begin{array}{l}\text { Spur winged } \\
\text { plover }\end{array}$ & In a hole in cultivated land \\
\hline Yellow wagtail & It nests in tussocks \\
\hline Icterine warbler & in a tree or a bush \\
\hline Whinchat & It nests in dense low vegetation \\
\hline Nightingale & $\begin{array}{l}\text { on the ground within or next to } \\
\text { dense bushes }\end{array}$ \\
\hline $\begin{array}{l}\text { Thrush } \\
\text { Nightingale }\end{array}$ & It nests low in dense bushes. \\
\hline
\end{tabular}

Trees: field study showed that all types of trees (e.g. Eucalyptus lastic, Morus alba and Phoenix dactylifera) were beneficial to birds.

Shrubs: shrubs and bushes were also suitable bird shelters, particularly for smaller birds or ground-dwelling species such as doves and quail.

Grass: field observations showed that long, ornamental grasses were ideal for small birds and ground-feeding species

Tree Cavities: the study showed that healthy, living trees were good shelter, but dead, hollow trees were also essential for many cavity-nesting birds. These cavities were also useful for roosting owls and winter bird shelters.

Birds and wildlife have adapted to utilize native plants that provide food (directly and indirectly), cover, nesting sites, shelter and protection, nesting material or a combination of resources, also birds provide plants with a number of benefits as birds help plants in seed dispersal, pollination and protection.

The avian community of Damietta coastal area may be classified into four types according to type of feeding: twenty of carnivores species, twenty one of insectivorous species, twenty one of omnivores species and four species of herbivores in a percent of $30.30 \%, 31.82 \%$, $31.82 \%$ and $6.06 \%$ respectively

Field study showed that plants rely on birds to disperse their seeds. Some species of bird are known to include some fruit in their diet. Even primarily insectivorous species such as Egyptian barn swallow and House sparrow will occasionally take small fruits. This is perhaps a result of the broad individual niches of the species in our relatively depauperate avifauna. Among the birds which eat fruits were Common bulbul and Hoopoe, these species chew and crack larger seeds with their relatively powerful bills and so must be regarded primarily as seed predators. Seeds of most fruits are indigestible and are eaten by animals and birds who deposit those seeds in their fecal matter. Since these creatures do not stay in the same place, the seeds are dispersed over a large area usually similar to that of where the mother plant thrived. They also have the benefit of being fertilized.

The relationships differed at the spatial and temporal scales, which made interpretation difficult, but the variations were possibly due to seasonality in migrants and phenological events of plant species [24]. However, previous specieslevel analysis showed that some habitat specific species are more prevalent only in the specific habitats [25]. Of the some 10,000 living bird species on earth [26], one in eight is threatened with global extinction [27]. Agricultural expansion and land use changes are leading drivers in the global decline of biodiversity $[28,29]$ and in the decline of about $60 \%$ of the birds listed on the IUCN (International Union for Conservation of Nature) Red List [28]. 
Human activities can alter the biota of ecosystems via extinctions and invasions [30,31]. It can greatly influence the structure of bird and plant communities. As areas are cleared due to agriculture, industry, urbanization and uprooting of Phoenix dactylifera. The vegetative structure of the habitat often undergoes major change, typically increasing in patchiness and decreasing in plant-species diversity [32]. Also, replacing native vegetation with introduced species decreases the foliage-height diversity and biomass of plants. As usable vegetative areas diminish, food availability to birds and the number of suitable breeding areas are reduced. Avian-species diversity typically drops with increasing disturbance, partly for these reasons [33].

On the basis of the present study we may conclude that birds are excellent measure of the health of the environment and of the sustainability of human progress. They occur in many habitats, can reflect changes in other animals and plants, and can be sensitive to environmental changes. A great deal of high quality data already exists, and new data are realistic and relatively inexpensive to collect. In addition, birds have meaning, resonance and symbolic value for many audiences, and are extremely popular with the public. The obtained information about the biotic relations between plants and birds may be useful for credible conservation, management of biodiversity and establishment of a nature reserve.

\section{References}

[1] S.M. Goodman, P.L. Meininger, The birds of Egypt. Oxford, UK, Oxford University Press. (1989)

[2] S.E. El-Kassas, Effect of some growth regulators on the yield fruit quality of Zaghloul date palm. Third Symposium on date palm. Date palm Research Center, King Faisal Univ., Saudi Arabia, 1 (1993) 179-186

[3] G. Shelly, A handbook on the birds of Egypt. John Van Voorst, London (1872)

[4] R. Meinertzhagen, Nicolls Birds of Egypt. Hugh Ress, London (1930)

[5] M.E. Tharwat, Birds known to occur in Egypt. Arabic Republic of Egypt, Cabinet of Ministers, Egyptian Environmental Affairs agency (EEAA), Department of Nature Protection, Publication of National Biodiversity Unit No. 8 (1997)

[6] A. El-Negumi, H. Zain El-Din, M. El-Momeiry, M. Fayed, Birds of Egypt. Dar El-Fikr El-Araby, Cairo. (In arabic) (1950)
[7] S. Goodman, Report on two small bird collections from Gabel Elbaregion, Southern Egypt. Bonn. Zool. Beitr. 35 (1984) 39-56

[8] Support for Environmental Assessment and Management (SEAM) Programme, Damietta Governorate, Environmental Profile, Ministry of State for Environmental Affairs, Egyptian Environmental Affairs Agency, Entec. Uk. Ltd., Erm, Uk, Department for International Development (2004)

[9] N.F. El-Arabany, S.E. Mansy, G.A. Abd-allah, S.E. Hassab El-Naby Comparisons between genetic and morphometric differentiation among populations of sparrows in Egypt. M. Sc. Thesis, Faculty of Science, Mansoura University, Egypt. (2007)

[10] R. MacArthur, J. MkcArthur, On bird species diversity. Ecology (1961) 594-598

[11] J. Finch, Family Obligations and Social Change. Cambridge, UK, Polity Press (1989)

[12] G.S. Mills, J.B. Dunning, J.M. Bates, The relationship between breeding bird density and vegetation volume. Wilson Bull 103 (1991) 468479

[13] M.S. Serag, Ecology of four succulent halophytes in the Mediterranean coast of Damietta, Egypt. Estuarina Coastal Shelf Sciences 49 (1999) 29-36.

[14] M.A. Zahran, A.J. Willis, The vegetation of Egypt, 2nd edn. Springer Science and Business Media B.V. (2009)

[15] M.S. Serag, An Ecological study on two geophytes: Asparagus Stipularis Forssk. and Asphoclelus aestivus Brot. In the Mediterranean coast of Egypt. Catrina 6 (2011) 47-58

[16] J. Braun-Blanquet, Pflanzensoziologie. Grundzüge der Vegetaionskunde. SpringerVerlag, Wien and New York. (1964)

[17] E.C. Pielou, Ecological Diversity. New York: Wiley. [A general book on ecological diversity. Entropy-based diversity measures. Models (for the distribution of species) (1975)

[18] V. Täckholm, Students' Flora of Egypt, 2nd edn. Cairo Univ. Publ., Cooperative Printing Company, Beirut, (1974) p. 888

[19] L. Boulos, Flora of Egypt Chicklist. Al-Hadara Publishing, Cairo, Egypt. (2009)

[20] E. Karen, E. Francl, D. Gary, relationships of human disturbance, bird communities, and plant communities along the land-water interface of a large reservoir. Environmental Monitoring and Assessment 73 (2002) 67-93

[21] F.C. James, N.O. Wamer, Relation between temporal forest bird communities and vegetation structure. Ecology 63 (1982) 159-171

[22] M.L. Morrison, T.A. Scott, Laying the foundation for a comprehensive program of restoration for wildlife habitat in a riparian floodplain. Environmental Management. 18 (1994) 939-955 
[23] D.T. Bolger, A.C. Alberts, M.E. Soule, Occurrence patterns of bird species in habitat fragment: sampling, extinction, and nested species subsets. American Naturalist 137 (1991)

\section{$96 \quad 155-166$}

[24] T.R. Shankar Raman, Effect of slash-and-burn shifting cultivation on rainforest birds in Mizoram, North-east India. Conservation Biology 15 ( 2001) 685-698

[25] N. Chettri, E. Sharma, D.C. Deb, Bird community structure along a trekking corridor of Sikkim Himalaya: A conservation perspective. Biological Conservation 12 (2001) $1-16$

[26] F. Gill, D. Donsker, (eds) IOC World Bird Names (version

2.6). http://www.worldbirdnames.org/. Accessed Nov., 2010

[27] J.E.M. Baillie, C. Hilton-Taylor, S.N. Stuart (eds) IUCN Red List of threatened species: A global species assessment. World Conservation Union (2004)

[28] K. Norris, Agriculture and biodiversity conservation: Opportunity knocks. Conserv Lett. 1 (2008) 2-11
[29] J.P.W. Scharlemann, R.E. Green, A. Balmford, Land-use trends in endemic bird areas: global expansion of agriculture in areas of high conservation value. Glob. Change Biol. 10 (2004) 2046-2051

[30] D.U. Hooper, F.S. Chapin, J.J. Ewel, A. Hector, P. Inchausti, S. Lavorel, J.H. Lawton, D.M. Lodge, M. Loreau, S. Naeem, B. Schmid, H. Setala, A.J. Symstad, J. Vandermeer, D.A. Wardle Effects of biodiversity on ecosystem functioning: A consensus of current knowledge. Ecological Monographs 75 ( 2005) 3-35

[31] M.A. Gray, S.L. Baldauf, P.J. Mayhew, J.K. Hill, The response of avian feeding guilds to tropical forest disturbance. Conservation Biology 21 (2007) 133-141

[32] D.A. Norton, R.J. Hobbs, L. Atkins, Fragmentation, disturbance, and plant distributions: Mistletoes in woodland remnants in the western Australian wheatbelt. Conserv. Biol. 9 (1995) 426-438.

[33] S.R. Beissinger, D.R. Osborne, Effects of urbanization on avian community organization. Condor 84 (1982) 75-83

تهاف الدراسة إلي إيجاد العلاقات الحيوية بين النباتات النامية بالمنطقة الساحلية لمحافظة دمياطو الطيور

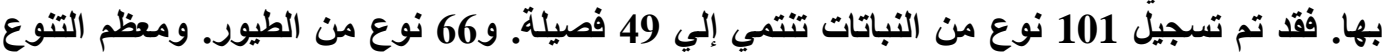

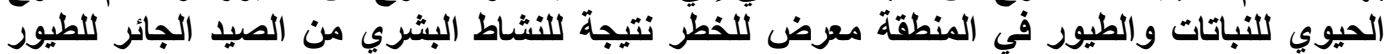

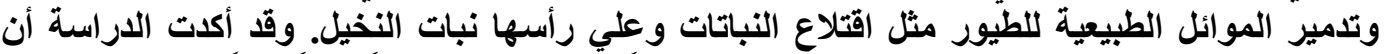

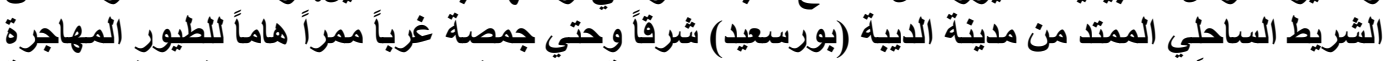

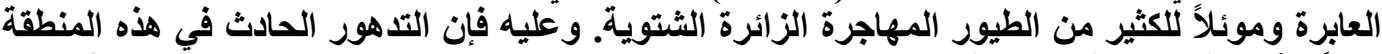

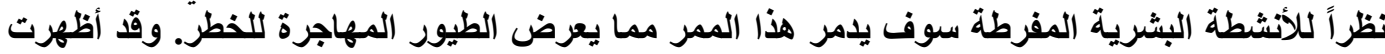

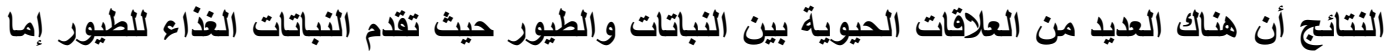

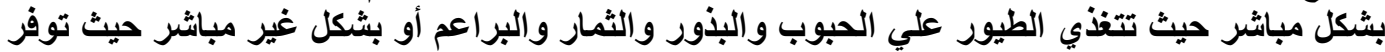

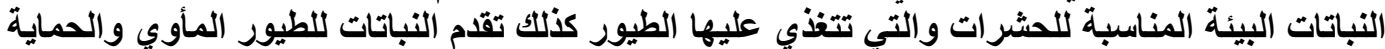

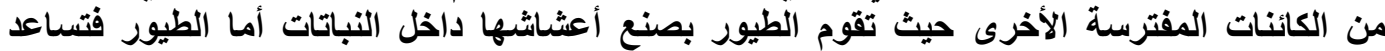

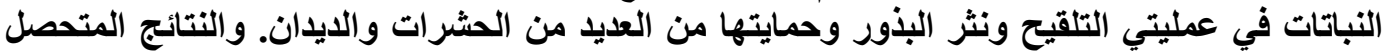

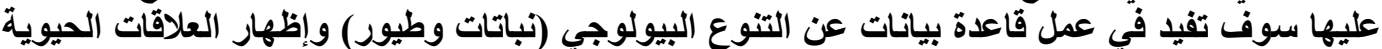

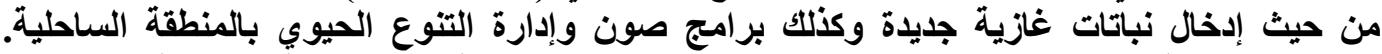

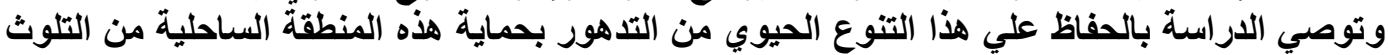
و إنشاء محمية طبيعية لصون الأنواع النادرة والمهددة بالانقر اض علي مستوي النباتات وكذلك الطيور. 